\title{
CAMBIOS FLoRísticos Y FISONÓMICOS DE LA VEgETACIÓN Y SU RELACIÓN CON LA CAPA CARBONATICO-CÁLCICA, EN ÁREA vitivinícola, Mendoza, Argentina
}

\author{
FLORISTIC AND PHYSONOMIC CHANGES OF VEGETATION AND ITS RELATIONSHIP \\ WITH THE CARBONATE-CALCIUM LAYER, IN THE VINEYARD AREA, MENDOZA, \\ Argentina
}

\author{
Mario Andrés Herrera Moratta*® , Ana Laura Navas Romero@ y \\ Antonio Daniel Dalmasso 10
}

Grupo de Geobotánica y

Fitogeografía- IADIZA - CONICET.

*maherrera@mendoza-conicet. gob.ar

\section{Citar este artículo}

HERRERA MORATTA, M. A., A. L. NAVAS ROMERO \& A. D. DALMASSO. 2019. Cambios florísticos y fisonómicos de la vegetación y su relación con la capa carbonatico-cálcica, en área vitivinícola, Mendoza, Argentina. Bol. Soc. Argent. Bot. 54: 241-254.

DOI: http://dx.doi. org/10.31055/1851.2372.v54. n2.24368

Recibido: 4 Julio 2018

Aceptado: 24 Abril 2019

Publicado: 30 Junio 2019

Editor: Guillermo Funes (iD

ISSN versión impresa 0373-580X

ISSN versión on-line 1851-2372

\section{SUMmaRY}

Background and aims: The province of Mendoza is characterized by a growing development of the cultivation of the vine. Its expansion has led to the occupation of areas with the presence of a layer of calcium carbonate. Our objective was to determine the depth of the "caliche" through the identification of plant communities and their zoning for use in the cultivation of the vine.

M\&M: The work was carried out in an area of 60 hectares. Twenty seven phytosociological surveys and 27 Point-quadrat transects were carried out in the field to determine the vegetation cover. In each the depth of the caliche was measured through the perforation technique and the hight of the dominant plant species were evaluated.

Results: There were 68 plant species recorded. The dominant matrix was represented by Larrea divaricata. Five floristic UV and physiognomic 5 UV were identified, finding a positive relationship between them. Caliche depth varied between $0-150 \mathrm{~cm}$. The floristic UV showed an overlap in the depth of the caliche, but not the physiognomic UV. Geoffroea decorticans and Senna aphylla were indicators of the presence of caliche.

Conclusions: The present work offers an alternative tool for the sampling of caliche depth, of low cost, high potential and effectiveness of use.

\section{KEY WORDS}

Caliche, cultivation, semiarid zone, vine.

\section{RESUMEN}

Introducción: La provincia de Mendoza se caracteriza por un creciente desarrollo del cultivo de vid. Su expansión, ha llevado a la ocupación de áreas con presencia de una capa de carbonato de calcio, el "caliche", planteando problemas agrícolas. Nuestro objetivo fue determinar la profundidad a la que se encuentra el "caliche" a través de la identificación de comunidades vegetales y zonificarlas para su uso con destino al cultivo de vid.

M\&M: Se trabajó en un terreno de 60 ha, Dpto. Tupungato. Se realizaron 27 censos fitosociológicos y 27 transectas de Point-quadrat para determinar cobertura vegetal. En cada punto de muestreo se midió la profundidad del caliche mediante la técnica de barrenado y se evaluó la altura de la vegetación dominante.

Resultados: Se registraron 68 especies vegetales. La matriz dominante estuvo representada por Larrea divaricata. Se identificaron 5 UV florísticas y 5 UV fisonómicas, encontrándose una relación positiva entre ambas. La profundidad de caliche osciló entre $0-150 \mathrm{~cm}$. Las UV florísticas mostraron un solapamiento en la profundidad del caliche, no así las fisonómicas. Geoffroea decorticans y Senna aphylla fueron indicadoras de la presencia de caliche.

Conclusiones: El presente trabajo ofrece una alternativa de bajo costo, alto potencial y efectividad de uso, para el muestreo de profundidad de caliche.

\section{Palabras clave}

Caliche, cultivo, vid, zona semiárida. 


\section{INTRODUCCIÓN}

La región del Oeste de la Argentina presenta características climáticas áridas y semiáridas que la hacen favorable para el desarrollo del cultivo de la vid. Dentro de la región, la provincia de Mendoza posee aproximadamente el $70 \%$ del área cultivada con vid y una producción de 65 $\%$ del total nacional, posicionándola en un lugar relevante para la agroindustria del vino (Agosta et al., 2012). Sin embargo, la continua expansión de esta actividad ha llevado a la ocupación desmedida de terrenos agrícolas, muchas veces con características topológicas no deseadas, como la presencia de "caliche" (capa de suelo de color grisáceo o crema que ha sido cementada por carbonatos de calcio y magnesio).

Abundantes regiones áridas y semiáridas presentan capas de "caliche" en el perfil del suelo que pueden presentarse como un horizonte suave y delgado; una capa dura y gruesa; o una capa expuesta a la superficie por erosión (Gile, 1961; SSSA, 2008). La presencia de suelo (cantidad de suelo bueno) por encima de un horizonte petrocálcico (capa de caliche) puede limitar drásticamente la producción de cultivos (Hamerlynck et al., 2002; Hennessy et al., 1983). Las capas de caliche muy duras plantean problemas en los cultivos agrícolas, impidiendo la expansión radical o generando asfixia por saturación hídrica. El caliche no endurecido no plantea problemas para el crecimiento y desarrollo de las raíces, pero puede imponer algunos problemas relacionados con la disponibilidad de nutrientes para el crecimiento de los cultivos, debido al $\mathrm{pH}$ y a la presencia de bicarbonatos (Hennessy et al., 1983; Schlesinger, 1982, 1985). En consecuencia, determinar la profundidad del caliche previo al cultivo de un suelo, se convierte en una actividad necesaria

En la actualidad, existen diversas técnicas que permiten su evaluación, sin embargo, la mayoría de ellas requieren abundante esfuerzo, tiempo y dinero.

Se conoce que los factores del paisaje juegan un papel importante en la organización de las comunidades vegetales (Rzedowski, 1956; Terradas, 2001; Whittaker \& Niering, 1965; Zavala-Hurtado et al., 1996). Esta correspondencia entre comunidades vegetales y suelos permitiría suponer que la heterogeneidad del ambiente edáfico controlaría en buena medida la distribución de las especies vegetales en el paisaje (Batista \& Taboada, 2005; Chaneton, 2005). En algunos casos el control edáfico operaría de modo directo, seleccionando especies tolerantes, pudiendo determinar extinciones locales e invasiones de especies vegetales que resultarán en un reemplazo dinámico entre diferentes tipos de comunidad (Batista \& León, 1992; Bochet et al., 2000; Sierra \& Perez, 2001). Es así que el empleo de especies y/o comunidades vegetales como indicadores de factores edáficos, como el caliche, se convierte en una estrategia plausible.

Basados en estos fundamentos y en la búsqueda actual de estrategias para la expansión de cultivos de vid, postulamos la hipótesis de que zonas con presencia de la capa de carbonato de calcio en superficie presentará diferentes asociaciones vegetales que zonas con capa de carbonato de calcio en profundidad. Por lo tanto, nuestro objetivo fue determinar las distintas profundidades a las que se encuentra el caliche (carbonato de calcio) a través de la identificación de caracteres florísticos y fisonómicos de las comunidades vegetales asociadas, y zonificar las mismas para su uso con destino al cultivo de vid.

\section{Materiales y Métodos}

\section{Área de estudio}

El área de estudio se ubica en el centro oeste de la Provincia de Mendoza (Argentina) distrito Gualtallary, Tupungato, en una zona rural al pie de la Cordillera de Los Andes. Posee una extensión de 60 ha de monte natural $\left(33^{\circ} 24^{\prime} 21,02^{\prime \prime}\right.$ Sur y $69^{\circ} 13^{\prime} 3,3^{\prime \prime}$ Oeste), a $1200 \mathrm{~m}$ s.n.m. El clima es templado con gran amplitud térmica diaria y estacional. La temperatura media anual es de $16,69{ }^{\circ} \mathrm{C}$, con una mínima absoluta de $-4{ }^{\circ} \mathrm{C}$ en el mes de julio y máximas absolutas de $42{ }^{\circ} \mathrm{C}$ en el mes de diciembre. Las precipitaciones anuales oscilan entre los 200 a $500 \mathrm{~mm}$ produciéndose principalmente entre los meses de primavera y verano (73\%) (Tonietto et al., 2012). Hay riesgos de heladas tardías con una probabilidad de ocurrencia del $10 \%$ en los meses de septiembre y octubre; mientras que existe riesgo de granizo durante la temporada de verano. La dirección 


\section{A. Herrera Moratta et al. - Cambios en vegetación y relación con la capa carbonático-cálcica}

dominante de los vientos es $\mathrm{O}-\mathrm{E}$ con velocidades de 5 a $7 \mathrm{~m} / \mathrm{s}$ (Llamas et al., 2013). Los suelos que dominan son una combinación de torrifluventes típicos con torriortentes muy pedregosos, con limitaciones por pendiente, presencia de caliche y con salinidad variable (Vargas Gil et al., 1990).

La vegetación pertenece a la Provincia Fitogeográfica del Monte, coincide con una estepa arbustiva arenosa con abundancia de Larrea divaricata, con un estrato herbáceo dominante de Nassella tenuis y Panicum urvilleanum (Furlani \& Gutierrez, 1996). El departamento tiene una especialización agrícola, los sistemas productivos de mayor desarrollo en la localidad son estacionales, con un importante desarrollo de la horticultura, fruticultura y, en los últimos años, un gran impulso de la vitivinicultura (Chazarreta $\&$ Bordón, 2010). La zona en estudio, posee un oasis con aproximadamente 4441 ha dedicadas al cultivo (Moreno, 2007). Esta localidad se encuentra conformada en su mayor parte por una gran extensión de grandes parcelas productivas con predominancia de vides finas con sistemas de riego presurizado.

\section{Evaluación de la vegetación Asociaciones vegetales}

Se trabajó sobre imágenes obtenidas de Google Earth (escala 1:2200) con una definición de 9 $\mathrm{m}$ e imágenes obtenidas a través de una cámara HERO5 Black de GoPro Dron (escala 1:2272) con una definición de $0,25 \mathrm{~m}$. El área de estudio se dividió en una matriz " $x$ " e " $y$ " en donde se seleccionaron de forma sistemática 22 puntos de muestreo. Además, se incorporaron 5 puntos de medición correspondiente a zonas de cauce. En cada punto de muestreo, se determinó la riqueza y cobertura vegetal mediante el uso del método de Point Quadrat (Daget \& Poissonet, 1971; Levy \& Madden, 1933; Passera et al., 1983). Cada medición consistió en 2 transectas perpendiculares de $30 \mathrm{~m}$ de largo cada una con intercepciones sistemáticas cada $0,3 \mathrm{~m}$. En la zona de cauce, las transectas se realizaron en forma paralela. Totalizando 54 transectas. Dicho valor de transecta es el recomendado para mediciones de Point Quadrat para matorrales de zona áridas (Dalmasso et al., 2017). En cada transecta se registró la identidad, número de especies, y número de contactos por especie. Para los nombres de las especies de plantas se utilizó la flora fanerogámica del Cono Sur (Zuloaga et al., 2013). Como la técnica de medición no es sensible a especies raras con frecuencias menores a 5, se acopló la medición con censos fitosociológicos para cada punto de muestreo (Roig, 1973). Con los datos obtenidos de los censos fitosociológicos y Point Quadrat se realizó un análisis de especies indicadoras de dos vías (TWINSPAN) para clasificar los relevamientos en diferentes grupos de composición florística (McCune \& Mefford, 1999). Para este análisis, se transformaron los datos de cobertura de PointQuadrat a la clasificación propuesta por BraunBlanquet (1932). Cada tipo florístico se nombró por el género de la especie con mayor cobertura promedio presente, seguido por el género de la segunda especie más abundante.

\section{Fisonomía de la vegetación}

En cada uno de los 27 puntos de muestreo, asociado a la información obtenida por medio de los datos de Point Quadrat se evaluaron diferentes medidas fisonómicas: cobertura vegetal total (\%), cobertura de árboles (\%), cobertura de arbustos (\%), cobertura de herbáceas (\%), altura máxima de la vegetación $(\mathrm{cm}) \mathrm{y}$, finalmente, la altura media de la vegetación $(\mathrm{cm})$. Cada tipo fisonómicos fue nombrado teniendo en cuenta los estratos más altos y dominantes. Para la determinación de la asociación fisonómica entre los puntos de muestreos se realizó un procedimiento de clasificación de K-medias (Jain \& Dubes, 1988). Simultáneamente en cada área evaluamos la altura de la especie vegetal dominante $(\mathrm{cm})$.

\section{Textura y Nivel hipsométrico}

En cada punto de muestreo geoposicionado ( 27 puntos de observación) se registraron los niveles hipsométricos, la latitud y la longitud con un GPS (Garmin eTrex 30).

Así mismo bajo cada punto de muestreo se recolectó una muestra de suelo entre $0-5 \mathrm{~cm}$ de profundidad totalizando 27 muestras. Cada muestra estuvo compuesta por tres submuestras abarcando así la heterogeneidad de la zona. Para la extracción se empleó un sacabocados metálico de $5 \mathrm{~cm}$ de diámetro. La textura fue determinada por la técnica de volumen de sedimentación (Nijenshon \& Maffei, 1996). 


\section{Evaluación de la profundidad del caliche}

En cada punto de muestreo geoposicionado (22 puntos de observación) se midió la profundidad del caliche mediante la técnica de barrenado, empleando un instrumento tipo zonda de acero pulido con mango de goma y punta cónica (largo: $1 \mathrm{~m}$, diámetro: $0,01 \mathrm{~m}$ ). Cada medición consistió en 5 barrenados, uno central y 4 equidistantes a $5 \mathrm{~m}$ del punto central. Efectuando un total de 110 barrenados. Esto permitió demarcar áreas con igual profundidad de la capa impermeable. En la zona de cauces ( 5 puntos de observación) no fue necesario realizar barrenado debido a que el caliche afloraba en superficie.

\section{Relación vegetación-profundidad caliche}

Con los datos de la vegetación se elaboró un cuadro sintético de relevamientos a los fines de identificar el comportamiento de la vegetación en función del medio físico, con énfasis en la presencia de caliche a distintas profundidades. A partir de ello se determinaron las unidades de vegetación y se destacó la presencia de especies nativas indicadoras de la capa impermeable. Analizamos la relación entre los grupos florísticos y los grupos fisonómicos a través de una tabla de contingencia. Sobre la base de esta tabla, realizamos una prueba de $\mathrm{X}_{\mathrm{i}}^{2} \mathrm{y}$ pruebas de bondad de ajuste (Agresti 2008) utilizando el paquete R 'stats' (R Foundation for Statistical Computing, Viena, AT). De esta manera, detectamos asociaciones significativas entre los grupos obtenidos con las dos clasificaciones.

Para conocer el porcentaje de predictibilidad de la profundidad del caliche en base a características superficiales (cobertura vegetal, tipo de suelo, fisonomía, ente otros) se utilizó un Modelo Lineal Generalizado (Nelder \& Wedderburn, 1972) con una distribución poisson, usando la función "glm" del paquete "RCMPR" (Ripley et al., 2015). La selección de los modelos se realizó siguiendo el criterio de información de Akaike (AIC) (Bolker et al., 2009). Antes del análisis estadístico, se comprobó que los datos cumplieran con las condiciones de sobredispersión de los datos residuales y homogeneidad de las varianzas con los gráficos de diagnóstico (qqnorm en R). Las variables independientes consideradas fueron: variables categóricas (3): Unidad Florística,
Tipo Fisonómico, Tipo de Suelo Superficial; variables cuantitativas (2): Cobertura Vegetal total, y Nivel de cota.

Con todos los datos obtenidos se llevó a cabo un proceso de superposición de información, identificándose áreas de solapamiento y aislamiento. Finalmente se construyó un mapa general, con las unidades de vegetación y la profundidad del "caliche". Todos los análisis estadísticos se realizaron utilizando el programa Insfostat (Di Rienzo et al., 2012). Para la delimitación de las unidades florísticas y fisonómicas se utilizó el programa Google Earth v7.3.1 (Google LLC, Mountain View, CA, USA). Para pasar las imágenes de “.kmz" a extensión ".shp" se utilizó el programa Global Mapper 16.0.5 (Blue Marble Geographics, Maine, Estados Unidos). Para la confección de mapas se utilizó el software QGIS v2.18.7 (Las Palmas de Gran Canaria).

\section{Resultados}

\section{Evaluación de las comunidades vegetales}

Se registraron un total de 68 especies vegetales. La matriz dominante estuvo representada por un Jarillal de Larrea divaricata. La presencia de un suelo arenoso y arenoso pedregoso, determinó un grupo de especies subdominantes, que, según las variaciones edáficas, podrían pasar a ser dominantes generando mosaicos diversos (Anexo 1).

El análisis de TWINSPAN permitió identificar 5 unidades florísticas: Schinus-Hyalis; LarreaGeoffroea; Larrea-Panicum; Larrea-Bougainvillea; y Larrea-Pappostipa (Fig. 1).

Schinus-Hyalis: Matorral húmedo dominado por Schinus fasciculatus y Hyalis argentea var. latisquama, acompañados por Larrea cuneifolia, Parkinsonia praecox ssp. glaucum, Sporobolus cryptandrus y Opuntia sulphurea. 28 especies presentes en un total de 5 puntos de relevamientos florísticos. Cobertura vegetal del $65 \%$.

Larrea-Geoffroea: Matorral de Larrea divaricata y Geoffroea decorticans acompañados por un estrato arbustivo bajo de Acantholipia seriphiodes, Descuriana canescens, Grindelia pulchella, Lycium chilensis y Pappostipa speciosa. Es una zona con una cobertura vegetal del $73 \%$ con 42 especies presentes en 3 relevamientos florísticos. 


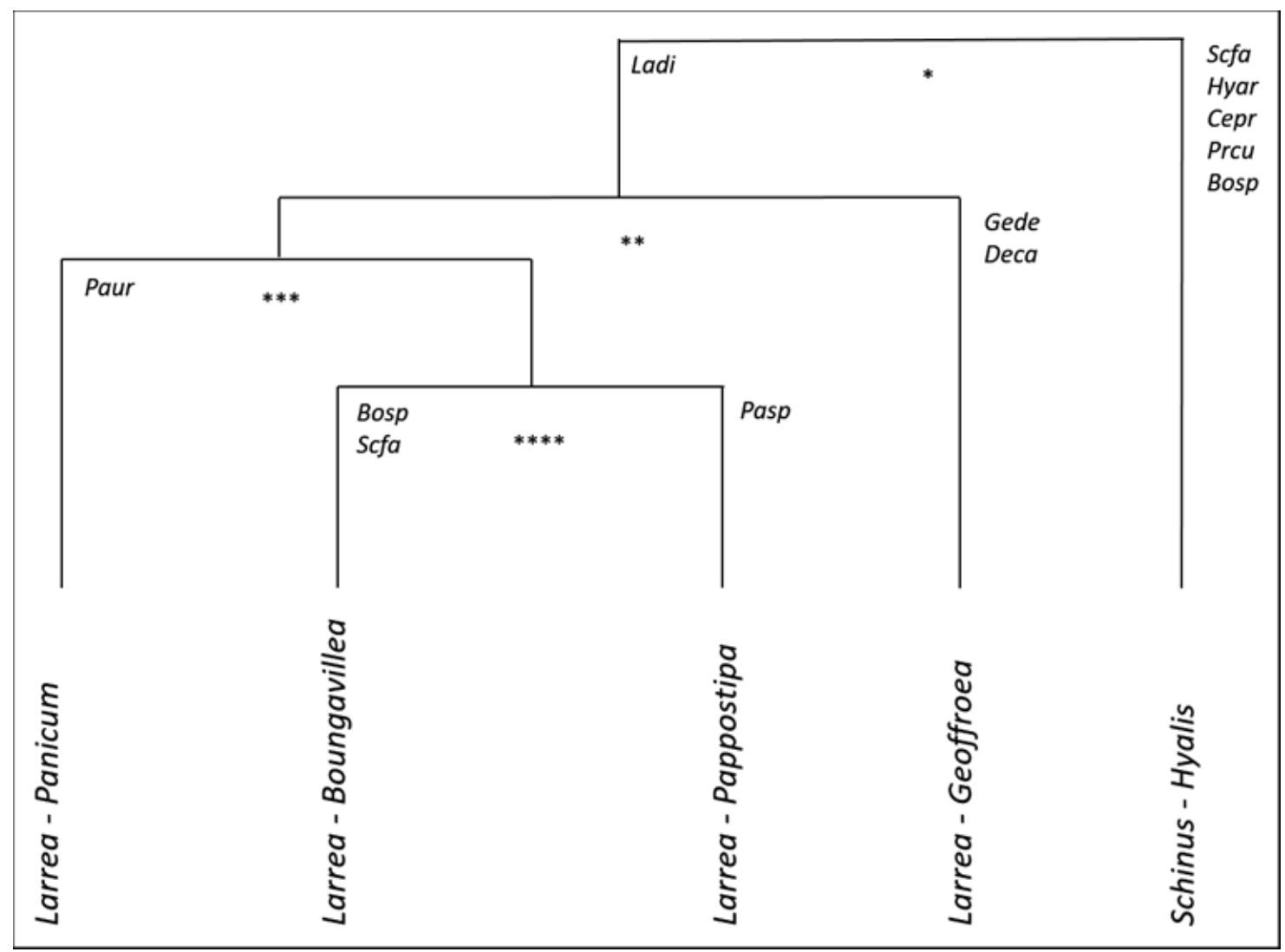

Fig. 1. Cladograma a partir de TWINSPAN de asociaciones florísticas. Cada uno de los censos de vegetación se agrupo de acuerdo a las interacciones positivas y negativas de las especies. En la división 1, la presencia de Ladi y Pasp con un factor de 0,28 fue la interacción de la distinción. En la división 2, la presencia de Gede y Deca $(0,164)$. En la división 3, la presencia de Paur $(0,165)$. En la división 4, la presencia de Bosp, Scfa $(0,171)$.

Larrea-Panicum: Matorral denso de Larrea divaricata acompañado de Panicum urvilleanum y Poa lanuginosa, con una alta presencia de Acantholipia seriphiodes y Prosopis flexuosa. Dos especies vegetales únicas Pappostipa vaginata y Lepidium sp. Posee una alta cobertura vegetal del $87,5 \%$ con 52 especies vegetales en 4 relevamientos florísticos.

Larrea-Pappostipa: Matorral denso de Larrea divaricata y Pappostipa speciosa acompaña por Poa lanuginosa, Jarava neaei y Acantholipia seriphiodes. Posee cuatro especies vegetales únicas, Bouteloua aristidoides, Ligaria cuneifolia, Baccharis trimera y Pterocactus tuberosus. Cobertura vegetal del 85.6 $\%$ con 58 especies vegetales en 7 relevamientos florísticos.
Larrea-Bougainvillea: Matorral ralo de Larrea divaricata y Bougainvillea spinosa acompañado por Acantholippia seriphiodes y Poa lanuginosa. Posee dos especies vegetales únicas, Taraxacum officinalis y Aloysia gratissima. Cobertura vegetal del $71 \%$ con 58 especies vegetales en 8 relevamientos.

El análisis fisonómico del paisaje, también determinó 5 unidades fisonómicas de vegetación: Jarillal alto; Jarillal bajo; Jarillal ralo; Chañaral y Cauce. Siendo las unidades de mayor cobertura total el Jarilla alto y el bajo (90\%), y el de menor cobertura el Cauce. La mayor cobertura de arbustos la presentó el Jarillal alto, mientras que la mayor cobertura de herbáceas fue para el Jarillal bajo (Tabla 1). 
Unidad 1- Jarillal Alto: Esta zona presenta una cobertura media del $90 \%$. Se caracteriza por la presencia de ejemplares de Larrea divaricata alto (> 1,50 m de altura), acompañado de arbustos leñosos de gran porte como Parkinsonia praecox var glaucum, Junellia seriphiodes, y Acantholippia seriphiodes. La vegetación herbácea se caracteriza por la presencia de vegetación psamófila como Panicum urvilleanum, Poa lanuginosa e Hyalis argentea var. latisquama. Los suelos son arenosos y profundos, encontrándose el caliche por debajo de los $100 \mathrm{~cm}$ de profundidad. Esta zona comprende aprox. 26 ha.

Unidad 2 - Jarillal Bajo: Esta unidad posee un $90 \%$ de cobertura vegetal media. Se caracteriza por una dominancia física de Larrea divaricata de porte bajo $(0,6 \mathrm{~m}-0,8 \mathrm{~m}$ de altura), compartida con otras especies leñosas como Lycium chilense, Acantholippia seriphiodes y Junellia seriphiodes de similar porte. La vegetación herbácea se encuentra dominada por Poa lanuginosa, Nassella tenuis y Jarava neaei. Los suelos son principalmente arenosos pedregosos, encontrándose el caliche entre los $0,6 \mathrm{~m}$ y $0,8 \mathrm{~m}$ de profundidad. Esta zona comprende una superficie aproximada de $22 \mathrm{Ha}$.

Unidad 3 - Jarillal Ralo: Esta unidad presenta un $86 \%$ de cobertura vegetal, con baja cobertura del estrato arbustivo. Se caracteriza por la presencia de ejemplares de Larrea divaricata bajo $(0,6 \mathrm{~m}-0,8 \mathrm{~m}$ de altura) Se encuentra acompañada de especies leñosas como Prosopis flexuosa var depressa, Senna aphylla y Acantholippia seriphiodes. La vegetación herbácea se encuentra dominada por un pastizal Poa lanuginosa, Sporobolus cryptandrus y Grindelia pulchella. Los suelos son pedregosos arenosos, encontrándose el caliche entre los $0,40 \mathrm{~m}$ - 0,15m de profundidad, dicha capa de caliche presenta una baja resistencia y cementación. Esta zona comprende aproximadamente 12 ha.

Unidad 4 - Chañaral de Geoffroea y Senna: Esta unidad presenta un $84 \%$ de cobertura vegetal. Se caracteriza por la dominancia de especie leñosas como Geoffroea decorticans y Senna aphylla, acompañadas de ejemplares de Larrea divaricata ralos. La vegetación herbácea se encuentra dominada por Poa lanuginosa, Baccharis pingraea y Chenopodium papulosum. Los suelos son pedregosos, y la profundidad del caliche en los mismos se encuentra cercana a los $0,15 \mathrm{~m}$ de profundidad.

Unidad 5 - Vegetación de los cauces: El altepe (Proustia cuneifolia) es la especie característica de estos wadis. Acompañan especies como el molle (Schinus fasciculatus), Baccharis pingraea, chañar brea (Parkinsonia praecox var glaucum), olivillo (Hyalis argentea var. latisquama), el tupe (Panicum urvilleanum).

Se encontró una dependencia entre las unidades fisonómicas y las asociaciones florísticas $\left(\mathrm{Chi}^{2}\right.$ test $=33,526, p=0,006$ ). De las 25 celdas de las combinaciones de los cinco tipos fisonómicos y los cinco grupos florísticos resultantes el $52 \%$ estaba vacío. Indicando que cada unidad fisonómica podría estar relacionada con las asociaciones vegetales (Tabla 2). Esto indica que cada tipo florístico puede adoptar varias fisonomías, y cada tipo fisonómico puede incluir varios tipos florísticos.

\section{Evaluación de la profundidad del caliche}

La profundidad de caliche osciló entre los 0-150 $\mathrm{cm}$, mostrando un gradiente de continuidad en el terreno (Fig. 2). En general se encontró escasamente cementado, y con aspecto pulverulento, permitiendo que las leñosas, con su sistema radical profundo, lograran sobrepasar esta limitante, evidenciando rasgos morfológicos visibles.

Tabla 1. Valores medios de las variables de diagnóstico para cada tipo de fisonomía.

\begin{tabular}{|cccccc|}
\hline & & \multicolumn{3}{c|}{ Fisonomía } & \\
& Cauce & Chañaral & Jarillal bajo & Jarillal ralo & Jarillal Alto \\
Cob. Total (\%) & 68,00 & 84,00 & 90,00 & 86,00 & 90,00 \\
Cob, Árboles (\%) & 21,00 & 5,00 & 2,00 & 1,00 & 2,00 \\
Cob, Arbusto (\%) & 34,00 & 48,00 & 43,00 & 51,00 & 52,00 \\
Cob, Herbáceas (\%) & 49,00 & 46,00 & 54,00 & 46,00 & 45,00 \\
Alt, máxima (m) & 1,57 & 0,60 & 0,76 & 0,53 & 1,12 \\
\hline
\end{tabular}


M. A. Herrera Moratta et al. - Cambios en vegetación y relación con la capa carbonático-cálcica

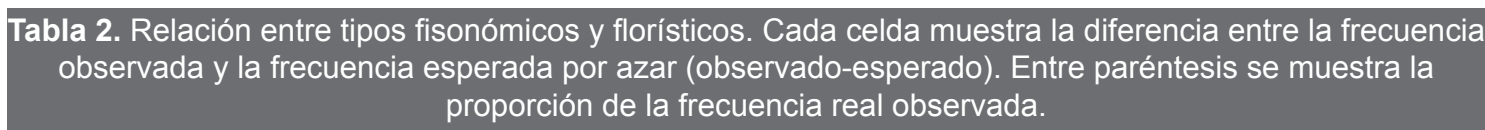

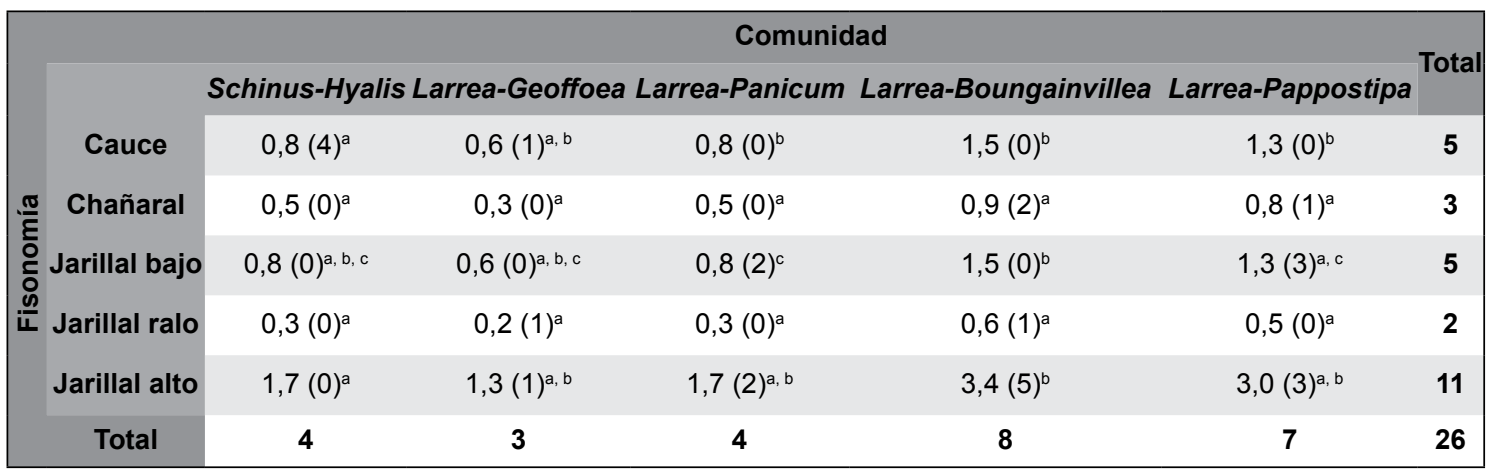

$\mathrm{Chi}^{2}$ test $=33,526, p=0,006$. Cada letra del subíndice denota un subconjunto de Comunidad. Categorías cuyas proporciones de columna no difieren de forma significativa entre sí en el nivel $(\alpha=0,05)$.

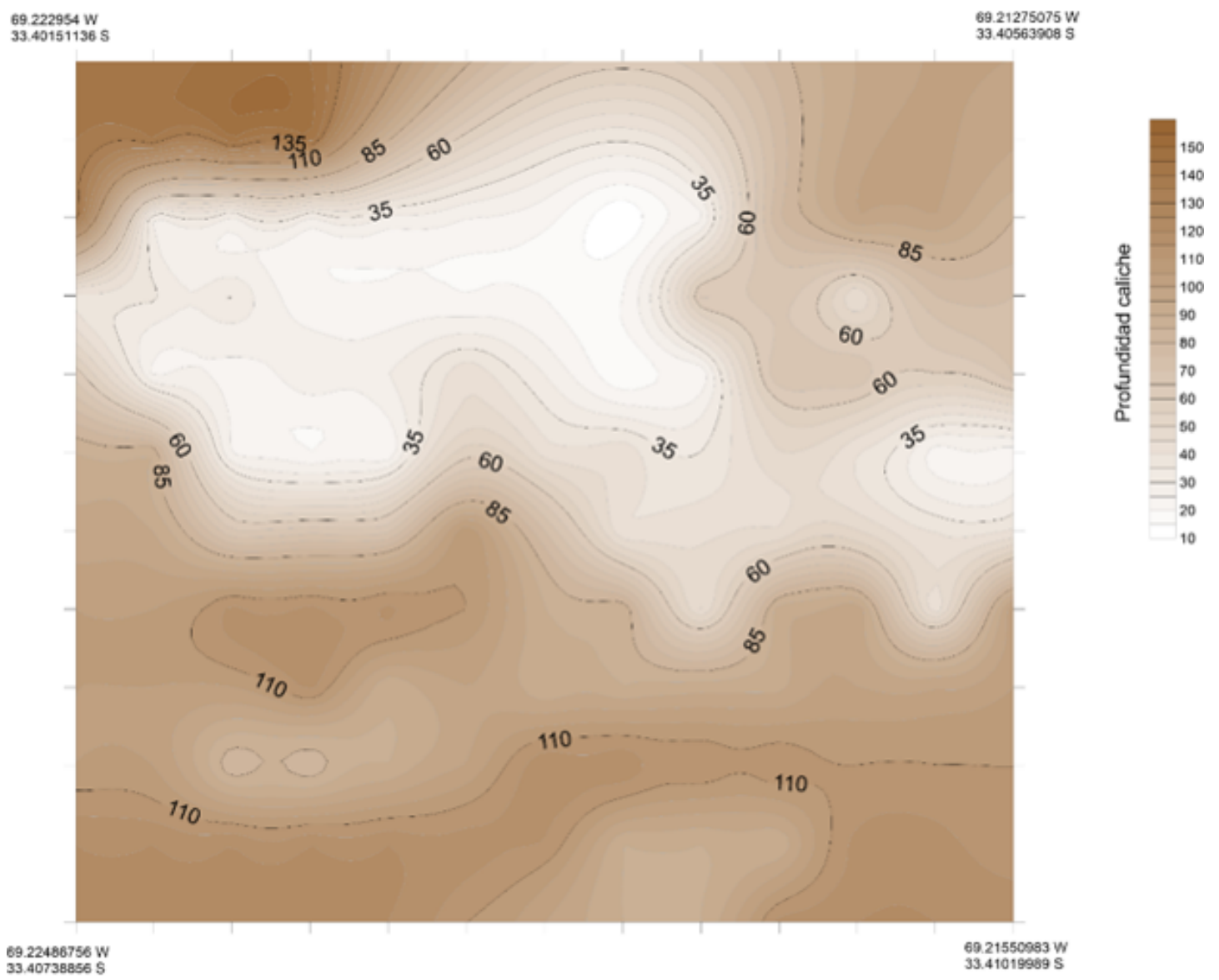

Fig. 2. Mapa de contorno de la profundidad del caliche. Las líneas de puntos indican zonas de igual profundidad. La escala se corresponde con colores más claros en zonas de menor profundidad a más oscuros en zonas de mayor profundidad de caliche. 
Las unidades florísticas mostraron en general un solapamiento en cuanto a la profundidad del caliche. La unidad Schinus-Hyalis, presentó una distribución limitada a su profundidad, aflorando el caliche en superficie ( $0 \mathrm{~m}$ de profundidad). La unidad Larrea-Geoffroea, aunque más amplia en su distribución, el caliche se ubicó entre los 0-27,5 cm de profundidad (Fig. 3a).

En el análisis fisonómico la relación entre profundidad del caliche y vegetación fue notable. La altura de la vegetación, así como la cobertura vegetal se correspondieron con la profundidad del caliche, siendo la fisonomía "Cauce" la más cercana a la capa impermeable, aflorando el caliche en superficie; y la fisonomía "Jarillal alto" la más lejana, ubicándose el caliche entre los 35$145 \mathrm{~cm}$ de profundidad. L. divaricata disminuyó su tamaño (de 1,50 a $0,80 \mathrm{~m}$ ) cuando la capa impermeable no superó $10 \mathrm{~s} 70 \mathrm{~cm}$ de profundidad; paralelamente se observó una reducción en la cobertura vegetal de la especie, con un menor número de ejemplares por superficie. Así en la fisonomía "Jarillal ralo" la profundidad del caliche se ubicó entre los $26-36 \mathrm{~cm}$ y en la "Jarillal bajo" entre los 19-66 cm (Fig. 3b).

De la misma manera, el modelo que mejor explicó la relación caliche con las variables independientes fue el que utilizó fisonomía de la vegetación, logrando explicar el 54,37\% de predictibilidad de la profundidad de la capa carbonatada (Tabla 3).

Las características de la comunidad vegetal, en cuanto a su cobertura y altura, fueron determinantes de los niveles de caliche. Sin embargo, las especies G. decorticans (chañar) y $S$. aphylla (pichanilla) fueron indicadoras de la presencia de una capa impermeable bajo la superficie. La cobertura de estas especies aumentó a un $10 \%$ respecto de la cobertura total, cuando el caliche afloraba hacia la superficie. El chañar formaba isletas de áreas localizadas (con raíces gemíferas), con un porte que no superaba los $2 \mathrm{~m}$ de altura.

Las especies herbáceas, específicamente las gramíneas perennes no fueron indicadoras de la presencia de la capa impermeable. Los suelos arenosos dominantes en el área fueron suficiente para la expresión de estas especies. En general, sólo las arbustivas de raíces profundas, mostraron diferencias en la morfología y la cobertura respecto de la capa impermeable, aunque la plasticidad adaptativa de muchas de ellas no permitió calificar con exactitud la variación del caliche.
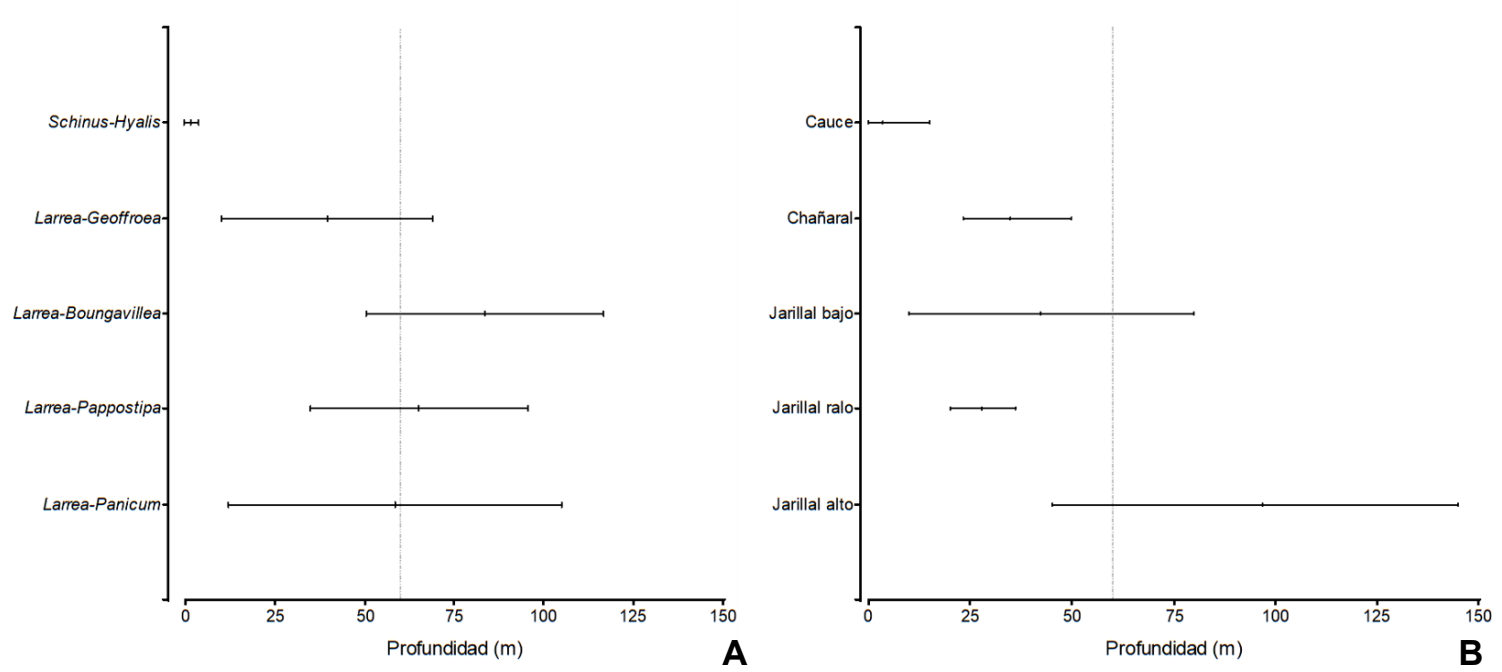

Fig. 3. Amplitud y profundidad media, indicadas por líneas verticales horizontales y cortas, respectivamente,a lo largo del gradiente de profundidad para cada tipo fisonómico (A) y florístico (B). 


\section{A. Herrera Moratta et al. - Cambios en vegetación y relación con la capa carbonático-cálcica}

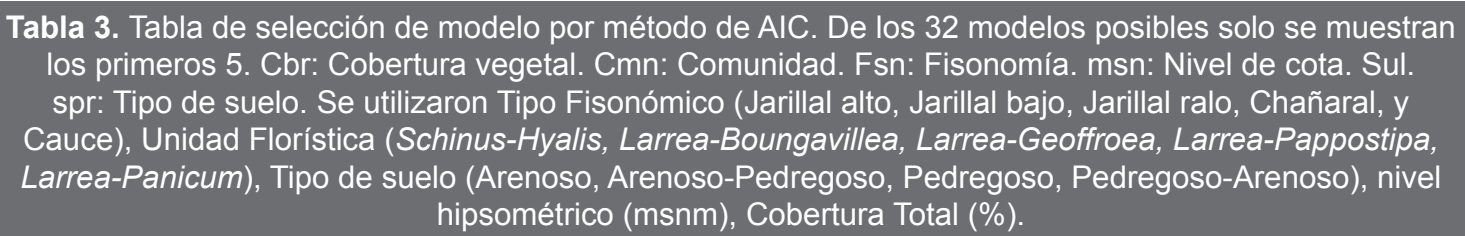

\begin{tabular}{|c|c|c|c|c|c|c|c|c|c|c|c|c|}
\hline & (Int) & Cbr & Cmn & Fsn & msn & Sul.spr & df & logLik & QAICc & delta & weight & \\
\hline 22 & 0,2615 & - & - & 0,4903 & - & + & 7 & $-98,944$ & 174,2 & 0,00 & 0,279 & $* * *$ \\
\hline 21 & 2,09 & 0,02341 & - & 0,5071 & - & + & 6 & $-101,909$ & 174,4 & 0,24 & 0,248 & ** \\
\hline 30 & $-26,91$ & 0,03006 & - & 0,4985 & 0,02164 & + & 8 & $-96,458$ & 175,2 & 1,02 & 0,168 & * \\
\hline 32 & $-32,46$ & 0,03181 & 0,168 & 0,5006 & 0,02551 & + & 9 & $-93,861$ & 176,7 & 2,51 & 0,08 & \\
\hline 24 & $-0,2968$ & 0,0237 & 0,139 & 0,492 & - & + & 8 & $-97,462$ & 176,7 & 2,54 & 0,079 & \\
\hline 23 & 1,519 & - & 0,1532 & 0,5074 & - & + & 7 & $-100,651$ & 176,7 & 2,58 & 0,077 & \\
\hline 29 & $-12,25$ & - & - & 0,519 & 0,01163 & + & 7 & $-101,344$ & 177,8 & 3,63 & 0,045 & \\
\hline 31 & $-16,97$ & - & 0,1762 & 0,5176 & 0,01495 & + & 8 & $-99,662$ & 180 & 5,87 & 0,015 & \\
\hline
\end{tabular}

Grado de significancia: ${ }^{* * *} 0,0001{ }^{* *} 0,001,{ }^{*} 0,01$.

\section{Discusión y Conclusiones}

La determinación de la profundidad del caliche es fundamental para disminuir pérdidas económicas en el productor. Las técnicas que emplean georadar, conductivímetros $\mathrm{y} / \mathrm{o}$ barrenada presentan complicaciones (Peralta et al., 2013; Vázquez et al., 2013). Para los primeros se requieren de largos periodos de calibración y puesta prueba en el terreno, siendo además el instrumental muy costoso y requiriendo de técnicos preparados. En los casos en los que se usa barrenado únicamente, si bien, el trabajo para medir la profundidad del suelo se realiza por única vez, la intensidad de trabajo que supone realizar los muestreos limita su uso a gran escala (Ross, 2012); requiriendo muchos meses con largas jornadas de trabajo. Gran parte del trabajo se facilita cuando se trabaja en áreas con homogeneidad de la vegetación, generando una zonificación que reduce la tarea. A partir de la zonificación y articulando la vegetación con el barrenado, fue posible caracterizar zonas con uniformidad en la distribución del caliche. Las unidades de vegetación desde el punto de vista fisonómico, respondieron a las condiciones edáficas (presencia de caliche). Asociaciones similares entre variaciones fisonómicas y características edáficas fueron detectadas por Galizzi et al. (1998) en donde el incremento en superficie calcárea se manifestó en una desaparición de especies como Stipa, Hordeum, y en un aumento de Bowlesia y Distichlis.

La cobertura y altura de las comunidades de Larrea se encontró fuertemente relacionada con la profundidad de la capa cementada, con una disminución en la cobertura y altura de Larrea con la reducción en profundidad de la capa cementada. De la misma manera De Marco et al. (1993), observaron que las comunidades de Larrea desaparecen cuando la capa es muy superficial y alcanzan sólo hasta $1 \mathrm{~m}$ de altura cuando la misma llega hasta $60 \mathrm{~cm}$ de profundidad. Por su parte Shreve \& Mallery (1933), encontraron que la profundidad del caliche se puede predecir con un elevado grado de precisión por la densidad y altura de los arbustos de Larrea, variando de 50 a $75 \mathrm{~cm}$ en los suelos más superficiales a 100 y $200 \mathrm{~cm}$ en los suelos profundos, siendo poco frecuente o ausente en áreas donde el caliche aflora en superficie. En suelos libres de caliche, Larrea alcanza mayor altura, ramifica más libremente, y soporta un follaje más pesado. Resultados similares fueron observados en Larrea tridentata por Hamerlynck et al. (2002; 2000) en el desierto de Mohave.

Estos ambientes son altamente selectivos y restringen el desarrollo de la vegetación a especies únicas, pastizales y/o comunidades mixtas de gramíneas con hierbas anuales, perennes y sufrútices 
(Gónzález-Medrano, 1996). En el área de estudio, las especies Geoffroea decorticans y Senna aphylla se comportaron como bioindicadoras expresándose cuando el caliche fue superficial. Las especies herbáceas perennes (hemicriptófitas y rizomatosas) respondieron a un suelo superficial arenoso, y no permitieron establecer diferencias respecto de la profundidad del caliche.

El presente trabajo ofrece una alternativa para el muestreo de profundidad de caliche, siendo una herramienta de bajo costo, expeditiva, alto potencial y efectividad de uso. Esta técnica permite a través del reconocimiento de comunidades claves mapear y detectar visualmente las capas cementadas. En definitiva, a través de la determinación de las comunidades y de sus atributos claves (como por ejemplo cobertura y altura) se puede mapear de manera más confiable zonas con diferentes profundidades de caliche. Lo que hace la técnica es facilitar y corroborar la altura del caliche, con menor esfuerzo de barrenado, permite delimitar en superficie, sin necesidad de hacer calicatas, y acelerar lo que ya de por sí es un procedimiento lento y tedioso. La mayor limitación que presenta es la imposibilidad de generalizar sobre cuáles son las comunidades que se corresponden con las diferentes profundidades de la capa impermeable, por lo que el estudio debe realizarse para cada zona en particular.

El manejo de suelos con caliche indurado depende en gran medida de la utilidad de la tierra. Para la producción comercial, es mejor evitar el uso de tierra con caliche poco profundo $(<1 \mathrm{~m}$ por debajo de la superficie) (Kirschbaum, 2000; Shreve \& Mallery, 1933). El caliche cementado limita el enraizamiento de los cultivos, y puede haber problemas con el anegamiento y la filtración lateral de los nutrientes, conduciendo a un mal rendimiento de los cultivos (Bautista-Zuñiga et al., 2004). El caliche, en general se encontró escasamente cementado, por lo que es factible su fracturación hasta los $0,40 \mathrm{~m}$ de profundidad con herramientas agrícolas.

Es importante, continuar este tipo de estudios para poder confirmar e identificar especies indicadoras de la profundidad del caliche y ajustar la técnica a cada terreno. En ocasiones se vuelve necesario realizar estudios mixtos con mapas de conductividad eléctrica, aunque estos presentan una elevada variabilidad espacial y en profundidad y muchas veces no coinciden con las profundidades del caliche (Vázquez et al., 2013), y otros sensores que permitan asociar los datos espaciales para determinar la profundidad y otras características del suelo de manera indirecta. Estos elementos pueden ayudar a determinar la variabilidad acorde al tipo de caliche (Ross, 2012).

\section{Contribución de los Autores}

Todos los autores han realizado conjuntamente y en partes iguales la colecta de datos, su interpretación y redacción del manuscrito.

\section{Agradecimientos}

Agradecemos al Tec. Darío Bustamante por su colaboración durante los muestreos en campo, a los encargados de la empresa Peñaflor por brindarnos las imágenes y a los revisores anónimos por los comentarios y sugerencias realizadas que ayudaron a mejorar sustancialmente el manuscrito. Este trabajo fue financiado por el Consejo Nacional de Investigaciones Científicas y Técnicas (CONICET) y la empresa Peñaflor.

\section{Bibliografía}

AgOSTA, E., P. CANZIANI, \& M. CAVAGNARO. 2012. Regional climate variability impacts on the annual grape yield in Mendoza, Argentina. J. Appl. Meteorol. Climatol., 51: 993-1009. https://doi.org/10.1175/JAMC-D-11-0165.1

AGRESTI, A. 2008. An Introduction to Categorical Data Analysis. Pharmaceutical Statistics (Vol. 7). https://doi.org/10.1002/pst.339

BATISTA, W., \& M. TABOADA. 2005. Asociación entre comunidades vegetales y suelos de pastizal de la Pampa deprimida. In Editorial. de la facultad de Agronomía (Ed.), La heterogeneidad de la vegetación de los agroecosistemas. Un homenaje a Rolando León. (pp. 113-129). Buenos Aires.

BATISTA, W.B., \& R.J. LEÓN. 1992. Asociación entre comunidades vegetales y algunas propiedades del suelo en el centro de la depresión del Salado. Ecol. Austral, 2: 47-55.

BAUTISTA-ZUÑIGA, F., H. ESTRADA-MEDINA, J. JIMÉNEZ-OSORNIO \& J. A. GONZÁLEZITURBE. 2004. Relationship Between Landforms and Soils in Karst Zones of Yucatán. TERRA Latinoam. 22: 243-254. 


\section{A. Herrera Moratta et al. - Cambios en vegetación y relación con la capa carbonático-cálcica}

BOCHET, E., J. POESEN, \& J.L. RUBIO. 2000. Moud development as an interaction of individual plants with soil, water erosion and sedimentation processes on slopes. Earth Surf. Process. Landforms 25: 847-867.

BOLKER, B.M., M.E. BROOKS, C.J. CLARK, S.W. GEANGE, J.R. POULSEN, M.H.H. STEVENS, \& J.S.S. WHITE. 2009. Generalized linear mixed models: a practical guide for ecology and evolution. Trends Ecol. Evol. 24: 127-135. https://doi.org/10.1016/j.tree.2008.10.008

BRAUN-BLANQUET, J. 1932. Plant sociology. The study of plant communities (1 ra ed.). McGraw-Hill Book Co., Inc., New York and London.

CHANETON, E.J. 2005. Factores que determinan la heterogeneidad de la comunidad vegetal en diferentes escalas espaciales. En: La heterogeneidad de la vegetación de los agroecosistemas. Un homenaje a Rolando J.C. León 90: 19-42.

CHAZARRETA, A.S., \& L.J. BORDÓN. 2010. Reestructuración productiva y transformaciones territoriales. Una aproximación empírica al departamento de Tupungato, Mendoza. Proyección (Aproximaciones teóricas, medio natural y transformaciones territoriales) 8: 141-165.

DAGET, P., \& J. POISSONET. 1971. Une méthode d'analyse phytologique des prairies. Critères d'application. Ann. Agron. 22: 5-41.

DALMASSO, A. 2010. Revegetacion de areas degradadas con especies nativas. Bol. Soc. Argent. Bot. 45: 149-171.

DE MARCO, G., F.A. ROIG, \& C. WUILLOUD. 1993. Vegetación del piedemonte andino en el centro oeste de Mendoza $\left(68^{\circ} 32^{\prime}-69^{\circ} 22^{\prime}\right.$ de Long. W y $33^{\circ} 42^{\prime}$ $34^{\circ} 40^{\prime}$ de Lat. S). Multequina 2: 201-241.

DI RIENZO, J., F. CASANOVES, M.. BALZARINI, L. GONZALEZ, M. TABLADA, \& C.. ROBLEDO. 2016. Infostat. FCA, Universidad Nacional de Córdoba, Argentina.

FURLANI, M.E., \& M.J. GUTIERREZ. 1996. Mendoza, una geografía en transformación (2 Edición). Mendoza: Universidad Nacional de Cuyo, Facultad de Filosofía y Letras.

GALIZZI, F., N. PEINEMANN \& S. LAMBERTO. 1998. Relación suelo-vegetación en áreas de transición entre condiciones de estepa y halomorfismo en el sur de la Provincia de Buenos Aires. Quebracho 8: 30-39.

GILE, L.H. 1961. A classification of Ca-horizons in soils of a desert region, Dona Ana County, New Mexico. Soil Sci. Soc. Am. 25: 52-61. https://doi.org/10.2136/ sssaj1961.03615995002500010024x

GÓNZÁLEZ-MEDRANO, F. 1996. Algunos aspectos de la evolución de la vegetación de México. Bol. Soc. Bot. México 158: 129-136. https://doi.org/10.17129/botsci.1493
HAMERLYNCK, E.P., J.R. MCAULIFFE, E. V MCDONALD, \& S.D. SMITH. 2002. Ecological responses of two Mohave desert shrubs to soil horizon development and soil water dynamics. Ecology 83: 768-779. https://doi.org/10.1890/00129658(2002)083[0768:EROTMD]2.0.CO;2

HAMERLYNCK, E.P., J.R. MCAULIFFE, \& S.D. SMITH. 2000. Effects of surface and sub-surface soil horizons on the seasonal performance of Larrea tridentata (creosotebush). Funct. Ecol. 14: 596-606. https://doi.org/10.1046/j.1365-2435.2000.00469.x

HENNESSY, J.T., J.M. GIBBENS, J.M. TROMBLE, \& M. CARDENAS. 1983. Water Properties of Caliche. J. Range Manag. 36: 6. https://doi.org/10.2307/3898195

JAIN, A.K., \& R.C. DUBES. 1988. Algorithms for clustering data. (N. Prentice-Hall, Inc. Upper Saddle River, Ed.). Michigan State University.

KIRSCHBAUM, M.U.F. 2000. Will changes in soil organic carbon act as a positive or negative feedback on global warming? Biogeochemistry 48: 21-51. https://doi.org/10.1023/A:1006238902976

LEVY, E.G., \& E.A. MADDEN. 1933. The point method of pasture analyses. New Zeal. J. Agric. 46: 267-279.

LLAMAS, S., I. TERESA, \& P. DANIEL. 2013. Reinserción de un antiguo basural para uso agrícola en Argentina. Rev. Int. Contam. Ambient. 29: 99-105.

MCCUNE, B., \& M.J. MEFFORD. 1999. PC-ORD: multivariate analysis of ecological data; Version 4 for Windows. MjM Softw. Des.

MORENO, M.S. 2007. Estudio comparativo. La situación del obrero rural temporario en dos modalidades productivas vitivinicolas diferenciales de Mendoza, ubicadas en las localidades de Isla Grande (Maipú), y Gualtallary (Tupungato). Universidad Nacional de Cuyo.

NELDER, J.A., \& R.W.M. WEDDERBURN. 1972. Generalized Linear Models. J. R. Stat. Soc. A. 135: 370-384. https://doi.org/10.1080/01621459.2000.10474340

NIJENSHON, L., \& J. MAFFEI. 1996. Estimación de la salinidad y otras características edáficas a través de los volúmenes de sedimentación. Cienc. Del Suelo 14: 119-121.

PASSERA, C.B., A.D. DALMASSO, \& J. BORSETTO. 1983. Método de Point Quadrat Modificado. Taller Arbusto Forrajeros Para Zonas Áridas y Semiáridas, 135-152.

PERALTA, N., J.L. COSTA, M. CASTRO FRANCO, \& M. BALZARINI. 2013. Delimitación de zonas de manejo con modelos de elevación digital y profundidad de suelo. Interciencia 38: 418-424.

RIPLEY, B.D. 2015. Choose a Model by AIC in a Stepwise Algorithm. $R$ Doc. 
ROIG, F.A. 1973. El cuadro fitosociológico en el estudio de la vegetación. Deserta 4: 45-67.

ROSS, F. 2012. Determinación espacial de la profundidad de suelo (tosca) y su relación con el rendimiento de cultivo. In In Congreso de Valor Agregado en Origen. 1. (pp. 1-3). Manfredi, Córdoba. Argentina.

RZEDOWSKI, J. 1956. Notas sobre la flora y la vegetación del estado de San Luis Potosí. III. Vegetación de la región de Guadalcazar. An. Inst. Bio. 27: 169-228.

SCHLESINGER, W.H. 1982. Carbon storage in the caliche of arid soils: a case study from Arizona. Soil Sci. 133: 247-255. https://doi.org/10.1097/00010694-198204000-00008

SCHLESINGER, W.H. 1985. The formation of caliche in soils of the Mojave desert, California. Geochim. Cosmochim. Acta 49: 57-66. https://doi.org/10.1016/0016-7037(85)90191-7

SHREVE, F., \& T.D. MALLERY. 1933. The relation of caliche to desert plants. Soil Science 35: 99-112. https://doi.org/10.1111/j.1365-2389.2008.01079.x

SIERRA, E.M., \& S.P. PEREZ. 2001. Rain ENSO in Junín, northern Buenos Aires, Argentina. Rev. Argent. Agrometeorol. 1: 51-57.

SOIL SCIENCE SOCIETY OF AMERICA. 2008. Glossary of soil science terms 2008. Soil science society of america, Inc. 93 pp.

TERRADAS, J. 2001. Ecología de la vegetación. (E. Omega, Ed.).
TONIETTO, J., V.S. RUIZ, \& V.D. GÓMEZ-MIGUEL. 2012. Clima, zonificación y tipicidad del vino en regiones vitivinícolas iberoamericanas. Programa Iberoamericano de Ciencia y Tecnología para el Desarrollo (1ra ed.). Madrid.

VARGAS GIL, J.R., J.P. CULTO, I. QUIROGA, E. CORVALÁN, J.J. NIEVA, P. NUÑEZ, \& G. CHACHAUA. 1990. Atlas de Suelos de la Republica Argentina. (INTA, Ed.), Cartographic Perspectives (Tomo II). Salta: Secretaría de Agricultura, Ganadería y Pesca. Proyecto PNUD ARG.

VÁZQUEZ, J.M., G. REPOSO, J.M. GUECAIMBURÚ, V. ROJO, \& C. GIACOBONE. 2013. Uso De Conductividad Electrica Del Suelo Para Determinar La Profundidad Al Horizonte Petrocalcico. Dep. Tecnol. Univ. Nac. Luján, 1-8.

WHITTAKER, R.H., \& W.A. NIERING. 1965. Vegetation of the Santa Catalina Mountains, Arizona: A Gradient Analysis of the South Slope. Ecology 46: 429-452. https://doi.org/10.2307/1934875

ZAVALA-HURTADO, J.A., P.L. VALVERDE, A. DÍAZ-SOLÍS, F. VITE, \& E. PORTILLA. 1996. Vegetation-environment relationships based on a life-forms classification in a semiarid region of tropical Mexico. Rev. Biol. Trop. 44: 581-590.

ZULOAGA, F.O., O. MORRONE, \& M.J. BELGRANO. 2008. Catálogo de las Plantas Vasculares del Conosur. Missouri Botanical Garden Press. 


\section{A. Herrera Moratta et al. - Cambios en vegetación y relación con la capa carbonático-cálcica}

Anexo 1. Cuadro sintético de relevamientos fitosociológico Pampa gualtallarí, La Tupungatita- Tupungato. Mendoza

\begin{tabular}{|c|c|c|c|c|}
\hline Suelo superficial & ArPed & ArPed & $\mathrm{Ar}$ & Ped \\
\hline Cobertura \% & 75 & 80 & 55 & 60 \\
\hline Estrato - Ambiente & Arb. & Cha. & Past. & Cau. \\
\hline Altura snm & 1204 & 1223 & 1242 & 1219 \\
\hline Unidades de vegetación & $\begin{array}{c}\text { Jarillal } \\
\text { L.divaricata }\end{array}$ & G. decorticans & $\begin{array}{c}\text { Panicum } \\
\text { urvilleanum }\end{array}$ & $\begin{array}{c}\text { P.cuneifolia-S } \\
\text { fasciculatus }\end{array}$ \\
\hline \multicolumn{5}{|l|}{ Especies } \\
\hline Larrea divaricata & III & 0.1 & II & 0.1 \\
\hline Prosopis flexuosa var. depressa & II & 0.1 & 0.1 & 0.1 \\
\hline Lycium chilense & I & 0.1 & . & 0.1 \\
\hline Condalia microphylla & 0.1 & 0.1 & . & 0.1 \\
\hline Acantholippia seriphioides & I & . & 0.1 & . \\
\hline Bougainvillea spinosa & 0.1 & 0.1 & 0.1 & 0.1 \\
\hline Geoffroea decorticans & 0.1 & IV & & 0.1 \\
\hline Senna rigida & 0.1 & $\mathrm{I}$ & 0.1 & 0.1 \\
\hline Nassella hialina & 0.1 & . & 0.1 & . \\
\hline Panicum urvilleanum & 0.1 & . & III & . \\
\hline Poa lanuginosa & 0.1 & . & 1 & . \\
\hline Pappostipa speciosa & 0.1 & . & 1 & 0.1 \\
\hline Nassella tenuis & 0.1 & . & 1 & . \\
\hline Junellia seriphioides & I & . & 1 & . \\
\hline Proustia cuneifolia & 0.1 & . & . & II \\
\hline Hyalis argentea var. latisquama & 0.1 & . & . & II \\
\hline Schinus fasciculatus & 0.1 & $\cdot$ & . & I \\
\hline Baccharis pingraea & 0.1 & 0.1 & . & I \\
\hline Parkinsonia praecox ssp. glaucum & 0.1 & . & . & 1 \\
\hline Acanthostyles buniifolium & 0.1 & . & $\cdot$ & . \\
\hline Lycium chilense var. minutifolium & 0.1 & . & 0.1 & . \\
\hline Grindelia chiloensis & 0.1 & . & $\cdot$ & . \\
\hline Hysterionica jasionoides & 0.1 & $\cdot$ & 0.1 & $\cdot$ \\
\hline Opuntia sulphurea & 0.1 & 0.1 & . & 0.1 \\
\hline Cereus aethiops & 0.1 & 0.1 & . & . \\
\hline Tagetes mendocina & 0.1 & . & 0.1 & 0.1 \\
\hline Conyza bonariensis & 0.1 & $\cdot$ & $\cdot$ & . \\
\hline Plantago patagonica & 0.1 & 0.1 & 0.1 & . \\
\hline Oenothera picensis & 0.1 & . & . & $\cdot$ \\
\hline Sporobolus cryptandrus & 0.1 & . & . & 0.1 \\
\hline Grindelia pulchella & 0.1 & 0.1 & . & 0.1 \\
\hline Ephedra ochreata & 0.1 & . & . & . \\
\hline Maihueniopsis darwinii & 0.1 & . & . & . \\
\hline
\end{tabular}


Bol. Soc. Argent. Bot. 54 (2) 2019

\begin{tabular}{|c|c|c|c|c|}
\hline Especies & & & & \\
\hline Bromus catharticus & 0.1 & . & 0.1 & . \\
\hline Glandularia radicata & 0.1 & . & . & . \\
\hline Heterotheca subaxillaris & 0.1 & . & . & . \\
\hline Lecanophora heterophylla & 0.1 & . & 0.1 & 0.1 \\
\hline Descurainia canescens & 0.1 & . & 0.1 & . \\
\hline Orobanche chilensis & 0.1 & . & 0.1 & . \\
\hline Echinopsis leucantha & 0.1 & . & 0.1 & . \\
\hline Salsola kali & 0.1 & . & . & . \\
\hline Philibertia gilliesii & 0.1 & . & . & 0.1 \\
\hline Baccharis darwinii & 0.1 & . & . & . \\
\hline Aristida mendocina & 0.1 & . & . & . \\
\hline Aloysia gratisima & 0.1 & . & . & 0.1 \\
\hline Thymophylla pentachaeta var. belenidium & 0.1 & . & . & . \\
\hline Thelesperma megapotamica & 0.1 & . & . & . \\
\hline Poa scaberula & 0.1 & . & . & 0.1 \\
\hline Chenopodium papulosum & 0.1 & . & . & . \\
\hline Solanum atriplicifolium & 0.1 & . & 0.1 & . \\
\hline Senna aphylla & 0.1 & . & . & . \\
\hline Setaria mendocina & 0.1 & . & . & 0.1 \\
\hline Trichocereus candicans & 0.1 & . & . & . \\
\hline Hoffmannseggia erecta & 0.1 & . & . & . \\
\hline Pyrrhocactus strausianus & 0.1 & . & . & . \\
\hline Baccharis trimera & 0.1 & . & . & . \\
\hline Verbesina encelioides & 0.1 & . & . & . \\
\hline Bouteloua aristidoides & 0.1 & . & . & . \\
\hline Digitaria californica & 0.1 & . & . & 0.1 \\
\hline Taraxacum officinale & 0.1 & . & . & . \\
\hline Solanum sp. & 0.1 & . & . & . \\
\hline Trichloris crinita & 0.1 & . & . & . \\
\hline Senecio filaginoides & 0.1 & . & . & 0.1 \\
\hline Calycera herbacea & 0.1 & . & 0.1 & 0.1 \\
\hline Ligaria cuneifolia & 0.1 & . & . & . \\
\hline Habranthus jamesonii & 0.1 & . & . & . \\
\hline Schismus barbatus & 0.1 & . & . & . \\
\hline Pterocactus tuberosus & 0.1 & . & . & . \\
\hline
\end{tabular}

Referencia: Arb. Arbustal; Ch. chañaral; Past. Pastizal rizomatoso; Cau. Cauce. 